\title{
Optimization of core phases of biolistic transformation of potato
}

\author{
Natalya P. Malakhova ${ }^{1,3}$, Yuriy A. Skiba ${ }^{1,2,3}$, Botakoz K. Tezekbayeva ${ }^{1,3}$, Elina $R$. \\ Maltseva ${ }^{1,2,3}$, and Gulnara A. Ismagulova ${ }^{1,2,3^{*}}$ \\ ${ }^{1}$ M. A Aitkhozhin Institute of Molecular Biology and Biochemistry, Laboratory of Plant \\ Bioengineering and Laboratory of Genome, 050012 Almaty, Republic of Kazakhstan \\ ${ }^{2}$ Almaty Branch of National Center for Biotechnology in Central Reference Laboratory (CRL), \\ 050054 Almaty, Republic of Kazakhstan \\ ${ }^{3}$ Institute of Plant Biology and Biotechnology, 050040 Almaty, Republic of Kazakhstan
}

\begin{abstract}
We have established the optimal exposure time for internodes and callus plant material of Aksor potato variety on the OSS osmotic medium before and after biolistics and the type of plant explant that is most suitable for that. The optimal objects were explants from internodes of test plants. The best time for exposure of plant material to the osmotic medium before bombardment is 24 hours, and after biolistics -48 hours. Callus tissue developed on the MS medium with zeatin two weeks later in internodes that were cultivated before bombarding on the OSS for 24 hours and we observed an increase in the callus mass by the end of fourth week. Not a single callus was obtained with rhizogenesis on the R4 medium from internodes and potato calli that were on the osmotic medium within 24 and 72 hours after biolistics. The regenerative capacity of internodes after cultivation on an osmotic medium for 48 hours after bombarding was higher than that of calli. We obtained 12 regenerant plants out of 200 internode explants and 4 regenerant plants out of 150 calli that were used in the experiment.
\end{abstract}

\section{Introduction}

The gene pool of potatoes (Solanum tuberosum) in the Republic of Kazakhstan consists of 1,450 samples from the worldwide collection from 32 countries and includes samples of wild and cultivated species, interspecific hybrids, as well as domestic and foreign varieties [1]. Due to its exceptional qualities, such as high yields, high content of carbohydrates, protein and vitamins, the demand for potatoes is constantly increasing compared to the other crops [2]. As one of the leading crops in agriculture, potatoes also serve as an important object for scientific research conducted in various areas of modern biotechnology. One of the pressing issues associated with the successful cultivation of potatoes, along with proper agricultural technology, is the use of varieties that are resistant to the action of various stress factors of abiotic and biotic nature.

Modern methods of plant genetic transformation make it possible to create plants that are resistant to pathogens in a short time, as well as having improved quantitative and qualitative

\footnotetext{
*Corresponding author: i_gulnara@mail.ru
} 
characteristics. Most experiments on biolistic transformation of potatoes are aimed at creating new lines with increased yield and resistance to fungal and bacterial pathogens. As a result, plants with an average increased yield of 50-80\% were obtained [3-5].

The individual characteristics of each plant species, namely, the ability to regenerate cells and the rate of response to damage during transformation, require the search for optimal conditions for each phase of gene transformation - from the choice of explant and to the exposure time after ballistics.

The objective of this research was to select plant explants for biolistic transformation of potato and to optimize the exposure time of material on the osmotic medium before and after bombarding.

\section{Materials and methods}

We used regenerated plants of potato variety "Aksor", which were obtained and recovered by the method of isolating apical meristems, as the research's objects.

Variety Aksor. The variety is mid-season, resistant for black scab, universal, potentially heat-resistant and drought-resistant. It has good storage quality and field resistance to viral diseases. It has a high starch content, which is up to $22 \%$. The variety is recommended for such regions as Almaty, Aktobe, Kyzylorda, Pavlodar, East Kazakhstan [6].

Callus induction was performed on the standard MS medium with addition of $30 \mathrm{~g} / \mathrm{l}$ of sucrose as a carbon source and 2,4-D as a hormone for initiating the callus production.

Preparation of callus tissue for biolistics was carried out on the OSS osmotic medium, the basis of which is the MS medium with a standard content of vitamins $-4.43 \mathrm{~g} / \mathrm{l}$, containing $60 \mathrm{~g} / \mathrm{l}$ of sucrose, $2 \mathrm{mg} / \mathrm{l}$ of 2,4-D and $36.43 \mathrm{~g} / \mathrm{l}$ of mannitol.

The transformation was carried out by the Biolistic Particle Delivery System PDS1000/He (Bio-Rad) ballistic installation with genetic constructs based on the pBI121 vector containing the kanamycin selective resistance gene under the control of the CaMV-35S promoter. Biolistics was carried out at a vacuum pressure of 91.4-94.8 $\mathrm{kPa}$, with gold nanoparticles bound to DNA according to the manufacturer's protocol. In order to conduct biolistics, we used $100 \mathrm{ng}$ of DNA (minimum expression unit).

After bombardment, the plant tissue was transplanted for recovery onto MS medium, containing $60 \mathrm{~g} / 1$ of sucrose. After recovery, the obtained transformed explants were planted on MS medium, containing zeatin at a concentration of $0.5 \mathrm{mg} / 1$, with the addition of the selective agent - kanamycin, at a concentration of $100 \mathrm{mg} / \mathrm{l}$. Selection went on from 45 to 65 days, after which Petri dishes with calli were exposed to the light in order to promote regenerants. Transformed plants were regenerated on the R4 medium, which is based on MS medium with a standard vitamin content of $4.43 \mathrm{~g} / \mathrm{l}$ and containing $30 \mathrm{~g} / \mathrm{lof}$ sucrose, $0.5 \mathrm{mg} / \mathrm{l}$ of zeatin and $2 \mathrm{mg} / \mathrm{g}$ of gibberellic acid.

\section{Results and discussion}

Among the biological parameters affecting the efficiency of transformation, the most important are the type of explants, their osmotic state, and the length of the incubation period on the culture medium before and after the bombarding. There is still no consensus among researchers regarding the timing of recultivation of explants before and after particle bombardment. These terms can vary greatly from one day to three weeks [7-8].

Preparation of explants for biolistics is about to increase the osmotic pressure in the nutrient medium by adding large amounts of sugar. The exposure of the explants on the osmotic medium allows them to better tolerate the vacuum conditions during the biolistic transformation. In addition, the features of the bombardment affect the placement of 
experimental material on a Petri dish. This is due to the fact that gold nanoparticles with deposited DNA disperse, covering only a certain area in the middle of the dish. Therefore, the material for transformation was placed tightly to each other in the middle of the Petri dish, as shown in Figure 1.

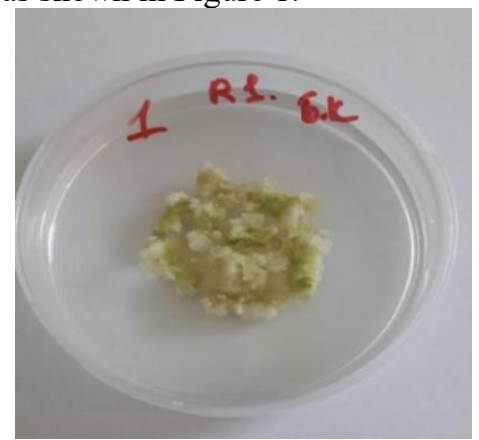

A

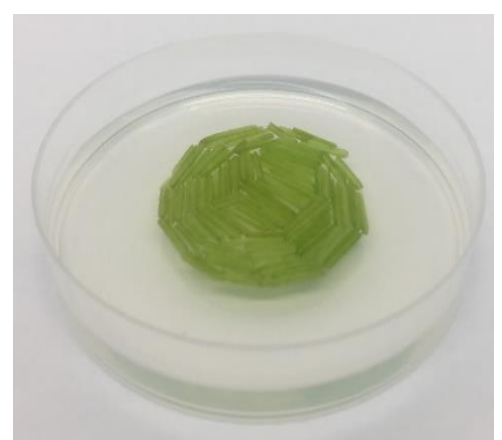

B

Fig. 1. Potato explants on the osmotic medium for biolistics. A - calli, B - fresh internodes.

The optimization phase included working out the required exposure time of plant material (internodes and calli of the test-tube potato plants) on OSS medium with mannitol and a standard vitamin content before and after biolistic transformation.

At the first stage, the optimal time for explants' location on OSS before ballistics was selected. In total, there were 3 options for duration of explants' location on the osmotic medium:

- Option 1: the explants were on the medium for 24 hours;

- Option 2: the explants were on the medium for 48 hours;

- Option 3: the explants were on the medium for 72 hours (Fig. 2, 3).

At the end of the cultivation time on OSS medium at the studied time intervals, the explants were transplanted onto MS medium with zeatin at a concentration of $0.5 \mathrm{mg} / \mathrm{l}$. The condition of the explants under study was assessed visually for four weeks from the day the experiment began, and the optimal exposure time was determined by the increase in the biomass of internodes and potato calli.

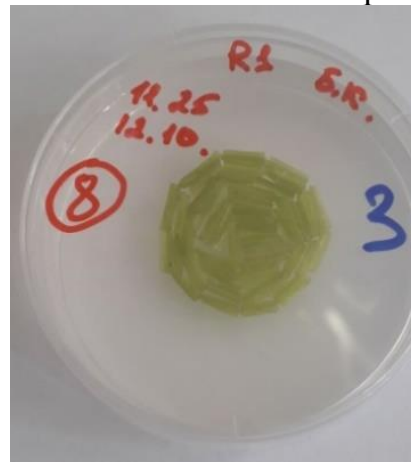

24 hours

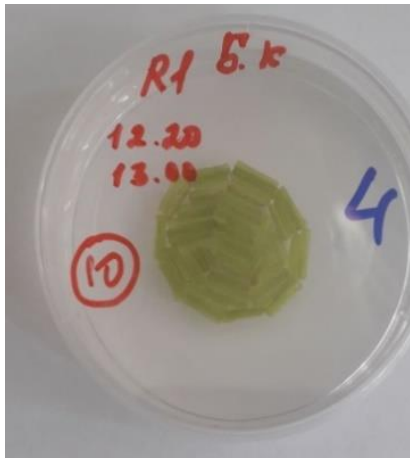

48 hours

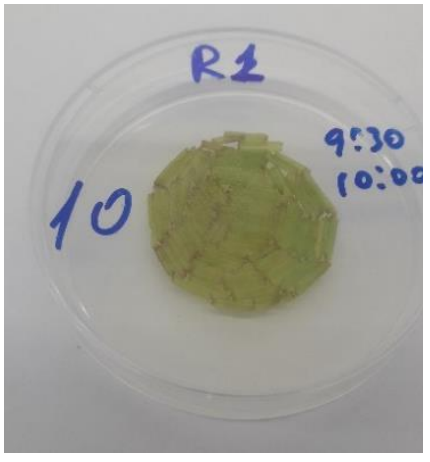

72 hours

Fig. 2. Potato internodes on the OSS medium before biolistics at various periods 


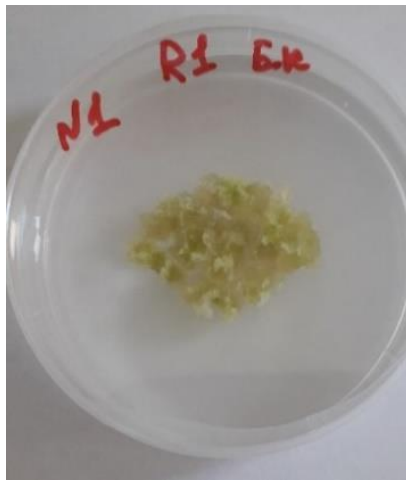

24 hours

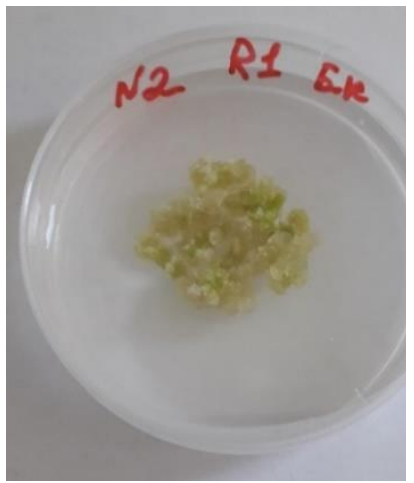

48 hours

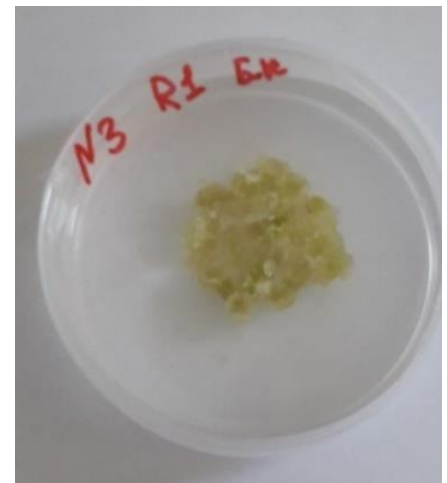

72 hours

Fig. 3. Potato callus explants on the OSS medium before biolistics at various periods

After two weeks of cultivating the internodes on MS medium with zeatin, callus tissue development was observed for 24 hours and by the end of the fourth week the formation of a morphogenic, dense, yellowish-green callus mass was observed. Potato calli under the same conditions became homogeneous and continued to increase mass.

The internodes located on the osmotic OSS medium for 48 hours also began to develop into callus tissue, but after four weeks, callus formation and increase in callus biomass were significantly lower. Callus cells, cultured on OSS for 48 hours, became loose and watery after four weeks.

Observations of internodes located on OSS for 72 hours showed that tissues gradually blackened upon further cultivation and the further growth of biomass stopped at the end of the experiment. Callus cells began to blacken and die faster than internodes explant under the same conditions (Figures 4, 5).

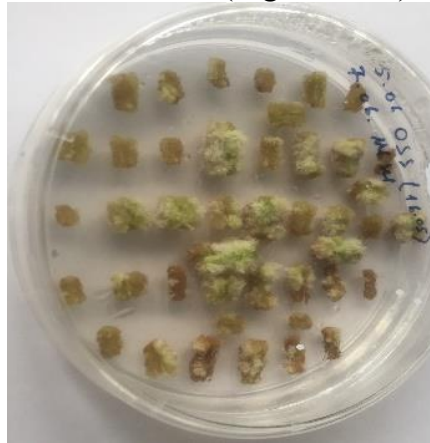

24 hours

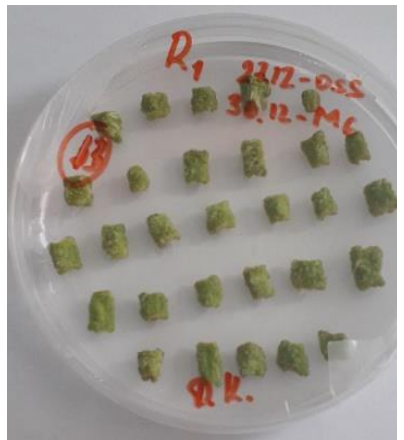

48 hours

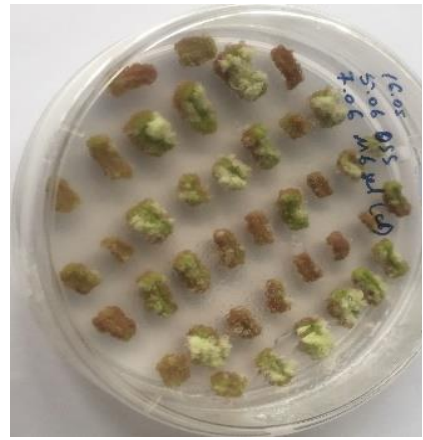

72 hours

Fig. 4. Internodes of Aksor potato varieties before biolistics in various time expositions 


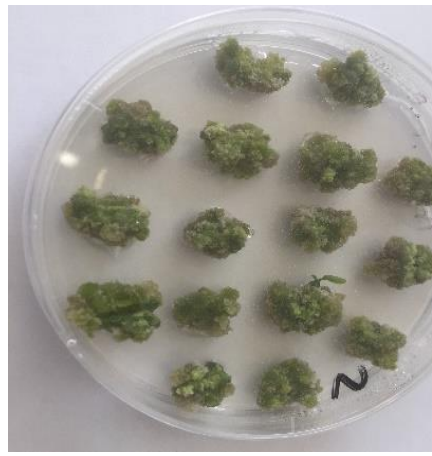

24 hours

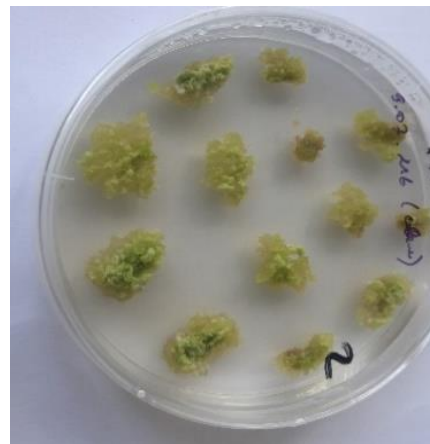

48 hours

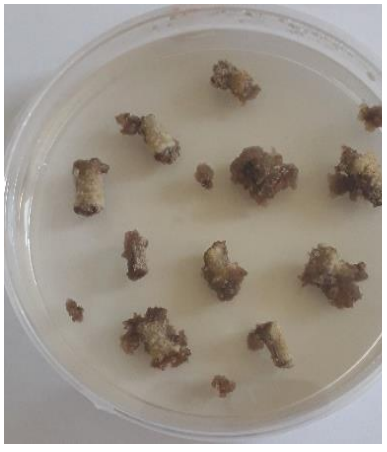

72 hours

Fig. 5. Calli of Aksor potato varieties before biolistics in various time expositions

It was established that option 1, namely, the cultivation of explants on an osmotic medium for 24 hours before biolistics, turned out to be the most optimal for further studies, because we observed increased formation of morphogenic callus.

The next phase of the research is the assessment of optimal time spent by explants on the OSS medium after biolistics. Based on the obtained experimental data of the first stage, the potato explants were kept on the OSS medium for 24 hours, after which biolistic transformation was carried out using a vector construct with the kanamycin resistance gene. As objects of transformation, we used one-day potato internodes that were cut lengthwise and laid on a $6 \mathrm{~cm}$ Petri dish with the cup side up and a 3 -week-old callus that was obtained from potato internodes on a medium for inducing callusogenesis with the addition of phytohormones 2,4-D $2 \mathrm{mg} / \mathrm{l}$ and zeatin $0.5 \mathrm{mg} / \mathrm{l}$.

To select the optimal cultivation time in OSS after biolistics, the explants were located in this medium for 24 hours (option 1), 48 hours (option 2) and 72 hours (option 3). The results of these experiments are presented in Figures 6 and 7.

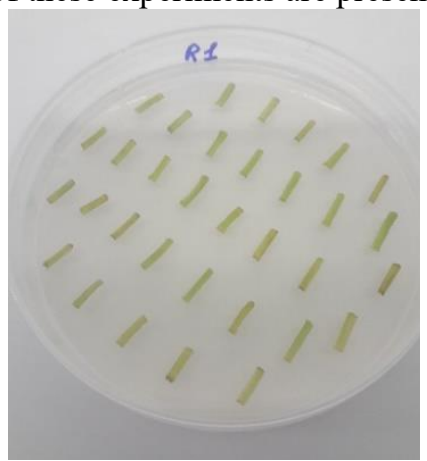

24 hours

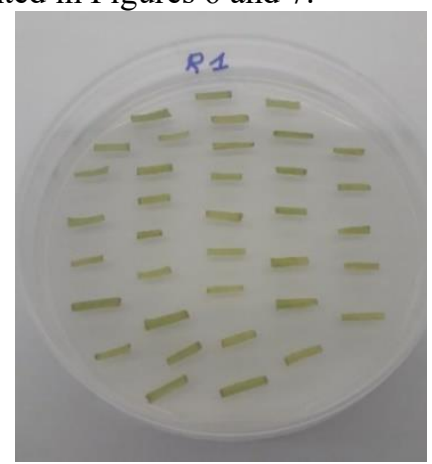

48 hours

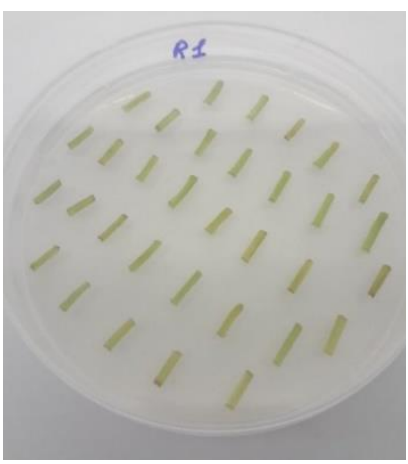

72 hours

Fig. 6. Potato internodes on the OSS medium after biolistics at various periods 


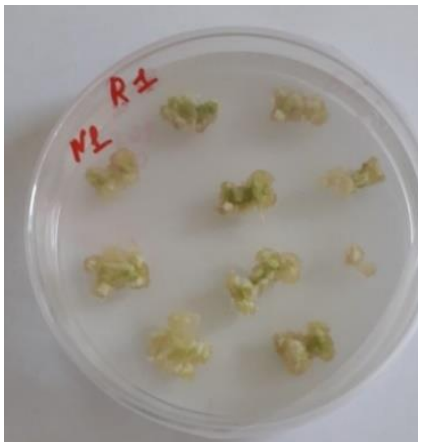

24 hours

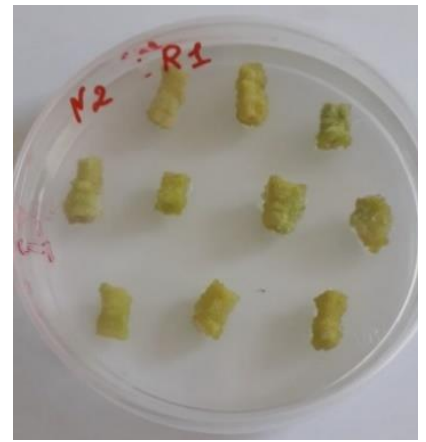

48 hours

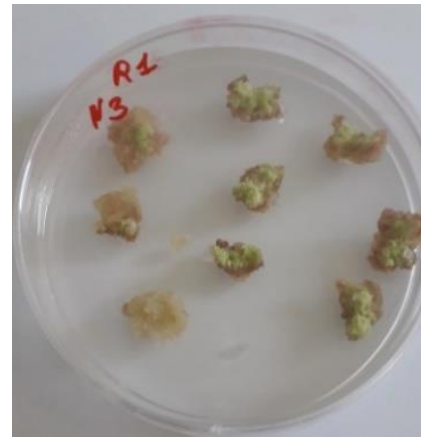

72 hours

Fig. 7. Potato callus explants on the OSS medium after biolistics at various periods

The optimal exposure time of the explants was estimated by the regenerative ability of potato internodes and calli upon their further cultivation on MS medium containing zeatin at a concentration of $0.5 \mathrm{mg} / \mathrm{l}$, with the addition of the selective agent - kanamycin, at a concentration of $100 \mathrm{mg} / \mathrm{l}$., Cells that carry the antibiotic resistance gene survived in the presence of a selective agent, while the untransformed cells died. The embryogenic calli with successfully visible selection signs of regeneration (seedling primordia) were selected for regeneration. Such calli were transferred onto the R4 medium containing zeatin $(0.5 \mathrm{mg} / \mathrm{l})$ and gibberelic acid ( $2 \mathrm{mg} / \mathrm{l})$ and which is used to stimulate the regenerative capabilities of the material. Potato explants actively formed seedlings in the presence of these hormones.

During the cultivation of explants on R4, it turned out that not a single callus with rhizogenesis was obtained from the potato internodes and calli that were on OSS medium within 24 hours after biolistics.

The regenerative capacity of internodes after cultivation on the osmotic medium for 48 hours after biolistics was high. In contrast to internodes, calli regenerated with less activity.

Explants located in this OSS medium for 72 hours after biolistics did not show regenerative ability, they blackened, and stopped their further growth by the end of the experiment (Figure 8, 9).

An analysis of the results showed that the optimal time for explants to be located on the OSS medium after biolistics is 48 hours. Explants that have been on this medium for more than 48 hours - within 72 hours after biolistics, did not show regenerative ability, as can be seen in Figures 8 and 9. It should be noted that internode explants showed greater regenerative ability in comparison with callus explants.

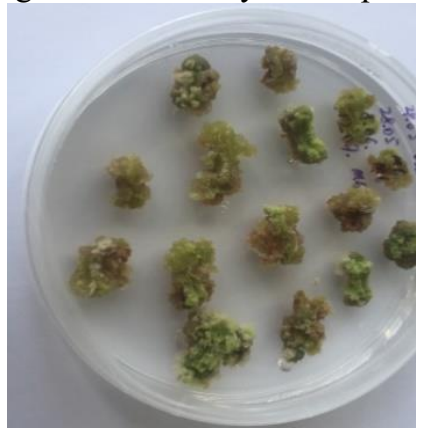

24 hours

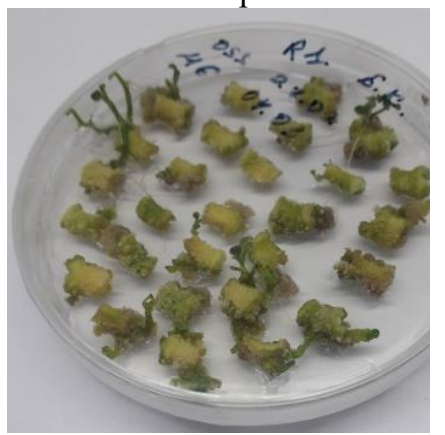

48 hours

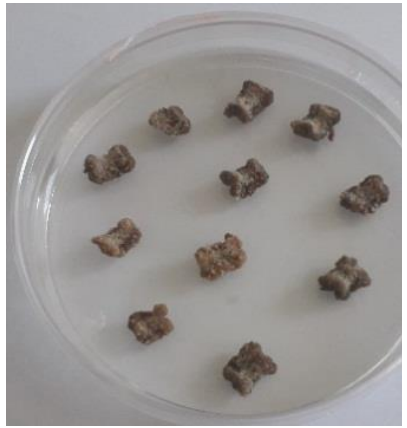

72 hours

Fig. 8. Regeneration of internodes of Aksor potato varieties after biolistics in various time expositions. 


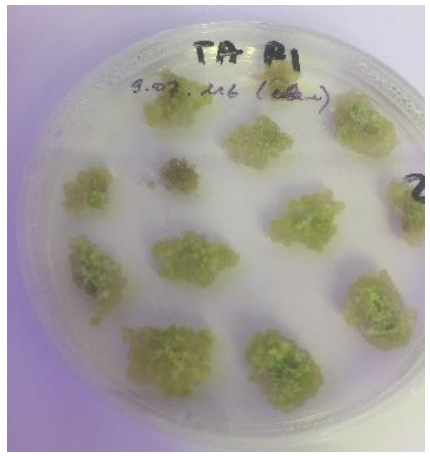

24 hours

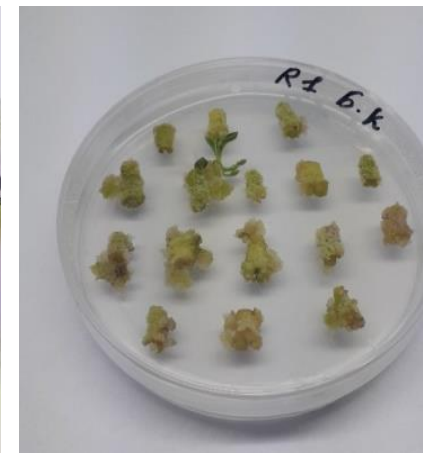

48 hours

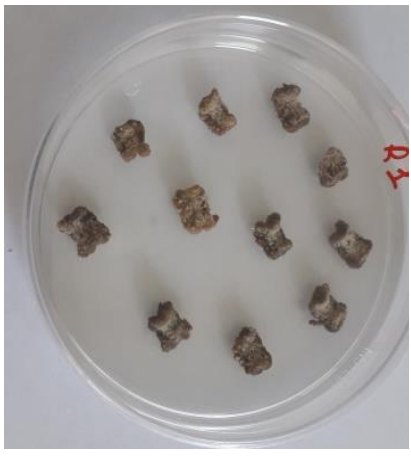

72 hours

Fig. 9. Regeneration of callus explants of Aksor potato varieties after biolistics in various time expositions

We transformed 200 internodes and 150 calli of the Aksor potato variety in total during the research. The number of potato regenerant plants after the transformation of various types of explants (internodes and calli) was significantly different. 12 regenerants were obtained out of the 200 internode explants, while the total yield of regenerated plants from 150 calli amounted to only 4 regenerants (Table 1).

Table 1. The number of potato regenerants after biolistic transformation on a selective medium

\begin{tabular}{|c|c|c|c|}
\hline Variety & Material & Explants amount & Regenerants amount \\
\hline \multirow{2}{*}{ Aksor } & internodes & 200 & 12 \\
\cline { 2 - 4 } & callus & 150 & 4 \\
\hline In total & & 350 & 16 \\
\hline
\end{tabular}

\section{Conclusion}

Hence, we selected and established the optimal exposure time of the plant material of internodes and calli of Aksor potatoes on the OSS osmotic medium before and after the biolistic experiment, and we determined the type of potato plant explant that is most suitable for biolistic transformation.

It has been established that the optimal explants with a high regeneration potential for carrying out the biolistic transformation of Aksor potatoes are the explants that are obtained from the internodes of test plants. The best exposure time of plant material of both internodes and potato calli on the osmotic OSS medium before and after the biolistics was 24 and 48 hours, respectively.

This research was carried out in the framework of the grant AR05136045 "New lines of wheat and potato created using a complex of innovational approaches of plant genetic engineering and cell technologies" and the targeted program BR05236574 "The development of advanced technologies to produce crops resistant to stress factors in utilizing adaptive mechanisms of plants", funded by the Ministry of Science and Education of the Republic of Kazakhstan in 2018-2020.

\section{References}

1. S. V. Kushnarenko, N. V. Romadanova, M. M. Aralbaeva, G. N. Matakova, M. O. Bekebaeva, D. I. Babisekova, Biotechnology. Theory and Practice 1, 28 (2013). DOI: 10.11134/btp.1.2013.6 
2. O. A. Fadina, M. P. Beketova, E. A. Sokolova, M. A. Kuznetsova, T. I. Smetanina, E. V. Rogozina, E. E. Khavkin, Agricultural Biology 52, 84 (2017). DOI: 10.15389/agrobiology.2017.1.84rus

3. X. Chen, D. Lewandowska, M. R. Armstrong, K. Baker, T.-Y. Lim, M. Bayer, B. Harrower, K. McLean, F. Jupe, K. Witek, A. K. Lees, J. D. Jones, G. J. Bryan, I. Hein, Theoretical and Applied Genetics 131, 1287 (2018). DOI: 10.1007/s00122-018-3078-6

4. N. A. Ryabushkina, N. N. Galiakparov, Biotechnology. Theory and Practice 3, 10 (2007)

5. B. Chakravarty, G. Wang-Pruski, B. Flinn, V. Gustafson, S. Regan, American Journal of Potato Research 84, 301 (2007). DOI: 10.1007/BF02986242

6. Aksor Kazahskij NII kartofelya i ovoshchebahchevyh kul'tur [Aksor Kazakh Research Institute of Potato and Vegetable Cultivars] (http://www.kartofel.org/cultivars/reg_cult/aksor.pdf)

7. F. Barro, A. Martin, P. A. Lazzeri, P. Barceló, Euphytica 108, 161 (1999)

8. M. Block, D. Debrower, T. Moens, Theoretical and Applied Genetics 95,125 (1997). DOI: $10.1007 / \mathrm{s} 001220050540$ 